\section{Ming-Mu Hsieh \\ Chien-En Hsu \\ Wei-Lung Tseng \\ Huan-Tsung Chang}

Department of Chemistry, National Taiwan University, Taipei, Taiwan

\title{
Amplification of small analytes in polymer solution by capillary electrophoresis
}

We report concentration methods for the analysis of small solutes by capillary electrophoresis in conjunction with laser-induced native fluorescence using a Nd:YAG laser. After injecting samples, poly(ethylene oxide) (PEO) in the anode reservoir entered a capillary filled with Tris-borate buffer. When migrating in PEO solution, the analytes slowed down and stacked at the interface between the sample zone and PEO solution. As a result, the limits of detection (LODs) down to 8 pm for 2-naphthalenesulfonic acid and $70 \mathrm{pm}$ for L-tryptophan have been achieved when injecting at $30 \mathrm{~cm}$ height for $120 \mathrm{~s}$ and $230 \mathrm{~s}$, respectively. Such low LODs are partially due to the effects of $\mathrm{NaCl}$ in the samples and PEO on the fluorescence characteristics of the analytes. In addition, the concentrations of $\mathrm{NaCl}$ and PEO have great impacts on the migration of the analytes and electroosmotic flow, thereby affecting resolution and speed. Without pretreatment, the determinations of five important markers in urine samples and two acids in a cerebrospinal fluid sample have been performed separately, with the relative standard deviations of the concentrations less than 3.6\%. Furthermore, by applying a short plug of low-pH buffer after injection, the analysis of greater volumes of the urine sample has been carried out, resulting in detecting more peaks.

Keywords: Capillary electrophoresis / Catecholamines / Laser-induced fluorescence / Polymer solution

\section{Introduction}

Following the success of the human genome project effort, a number of other "omic" disciplines have emerged, including proteomics, pharmaconomics, metabolomics, with a goal toward the analysis of the components of a living organism in its entirety [1-3]. In comparison to genomics, metabolomics, aiming to obtain a complete set of metabolites, is more difficult because its patterns change, depending on factors such as the stage of development of an organism and the organism's physiological states. Other difficulty is that their amounts cannot be amplified by polymerase chain reaction, thus a highly sensitive method is extremely needed. Besides, it is not easy to complete a set of metabolites because the determination of a vast number of small analytes, including organic and inorganic neutral molecules and ions, with

Correspondence: Dr. Huan-Tsung Chang, Department of Chemistry, National Taiwan University, Taipei, Taiwan, R.O.C.

E-mail: changht@ccms.ntu.edu.tw

Fax: +886-2-23621963

Abbreviations: 5-A-1-N, 5-amino-1-naphthol; 7-A-4-H-2-NSA, 7-amino-4-hydroxy-2-naphthalenesulfonic acid; CF, concentration factor; CSF, cerebrospinal fluid; 1,3-DHN, 1,3-dihydroxynaphthalene; 5-HIAA, 5-hydroxyindole-3-acetic acid; 5-HT, 5-hydroxytryptamine; HVA, homovanillic acid; 3-IXS, 3-indoxyl sulfate; 1-NAA, 1-naphthylacetic acid; 2-NSA, 2-naphthalenesulfonic acid; PEO, poly(ethylene oxide); TB, Tris-borate; TP, Tris-propanate; VMA, DL-vanillomandelic acid concentrations ranging several orders of magnitude may be required. Thus, techniques allowing sensitive and high-resolution analysis of small solutes are highly demanded.

Capillary electrophoresis (CE) has been often promised as a viable and easy-to-use tool for analysis of small solutes for more than two decades [4-6]. It, however, suffers from poor concentration detection due to a small sample volume and a short optical path length when utilizing any optical detection methods. For instance, the limits of detection (LODs) for common solutes are generally on the order of $\mu \mathrm{M}$ using UV-Vis absorption [7]. In order to improve sensitivity, laser-induced fluorescence (LIF) with tedious derivatization processes is commonly employed [8]. To prevent problems associated with derivatization, UV lasers have been used for the analysis of macromolecules and small solutes [9-13].

On-line concentration techniques are alternative for improving sensitivity, which can be promoted through differences in $\mathrm{pH}$, conductivity, salt content, viscosity, and additive concentration [14-17]. The focusing efficiency relies on specific modification of the composition of the electrolyte relative to the background electrolyte. Generally, it is much easier and straightforward for the concentration of analytes prepared in low-conductivity media than in high-conductivity media, because salt could cause Joule heating, loss of resolution and poor stacking $[18,19]$. However, in view of life science, techniques 
allowing on-line concentration of high-salt samples are more important, with regard to simplicity and ease of automation. Isotachophoretic (ITP) preconcentration method requires a leading buffer (at the detector side) and a terminating buffer (at the injection side) in two sides of the sample plug and has been applied to the analysis of analytes, with 100 to 1000 -fold improvements in sensitivity $[20,21]$. Acetonitrile-salt mixtures have been employed for the analysis of peptides in plasma, with at least a 20fold enhancement in sensitivity [22]. Another interesting example is that, after sample injection, an acidic or basic buffer was applied to generate $\mathrm{pH}$ changes for concentration of analytes prepared in high-conductivity media [23]. Concentration of small analytes has also been carried out in the presence of micelles that stack in the background electrolyte prior to interactions with neutral analytes prepared in high-salt media [24, 25]. Recently, dynamic $\mathrm{pH}$ junctions generated in a multisection electrolyte system have been demonstrated for concentration of small solutes [26, 27].

Herein, we developed a technique, similar to the one used for concentration and separation of macromolecules [2830], for the analysis of small solutes using polymer solutions. When migrating from the sample zone to polymer solutions, the solutes slowed down and thus stacked. In this study, our efforts were also made to explore factors, such as $\mathrm{pH}$, salt, and concentration of $\mathrm{PEO}$, on resolution, migration time and stacking. In addition, the developed method was applied to the determination of some important markers such as amine metabolites in urine and cerebrospinal fluid (CSF).

\section{Materials and methods}

\subsection{Apparatus}

The basic design of the separation system has been previously described [29]. Briefly, a high-voltage power supply (Gamma High Voltage Research, Ormond Beach, FL, USA) was used to drive electrophoresis. The entire detection system was enclosed in a black box with a high-voltage interlock. High-voltage end of the separation system was put in a home-made plexiglass box for safety. An $\mathrm{Nd}$ :YAG laser with $266 \mathrm{~nm}, 3000 \mathrm{~Hz}$ output from Continuum (model: EPO-5000; Santa Clara, CA, USA) was used for excitation. One UG 5 filter from Melles Griot (Irvine, CA, USA) was used to block $532 \mathrm{~nm}$ lights from the laser. The emission light was collected with a $10 \times$ objective (numeric aperture $=0.25$ ). One UG 1 filter from Edmund (Barrington, NJ, USA) was used to block scattered lights before the emitted light reached the photomultiplier tube (Hamamatsu R928). The amplified currents were transferred directly through a 10-k $\Omega$ resistor to a 24-bit A/D interface at $10 \mathrm{~Hz}$ (Borwin, JMBS Developments, Le Fontanil, France) and stored in a personal computer. Capillaries (Polymicro Technologies, Phoenix, AZ, USA), $75 \mu \mathrm{m}$ ID $\times 365 \mu \mathrm{m}$ OD, were used for the analysis of small solutes without any further coating process.

\subsection{Materials}

Human serum albumin (HSA) was from Sigma Chemical (St. Louis, MO). 5-Amino-1-naphthol (5-A-1-N), 1,3-dihydroxy-naphthalene (1,3-DHN), 1-naphthylacetic acid (1-NAA), 2-naphthalenesulfonic acid (2-NSA), 7-amino4-hydroxy-2-naphthalenesulfonic acid (7-A-4-H-2-NSA), L-tryptophan, 5-hydroxytryptamine (5-HT), 5-hydroxyindole-3-acetic acid (5-HIAA), homovanillic acid (HVA), DL-vanillomandelic acid (VMA), 3-indoxyl sulfate (3-IXS) and all chemicals for preparing buffer solutions were obtained from Aldrich (Milwaukee, WI, USA). TB buffers prepared from Tris were adjusted with boric acid to $\mathrm{pH}$ 10,9 , and 8 , respectively. Herein, $\times$ mM TB buffer means $\times \mathrm{mm}$ Tris solution adjusted with suitable amounts of boric acid. PEO $\left(M_{\mathrm{r}} 8000000\right)$ solutions were prepared in TB buffer, pH 9 [28]. The analytes were separately prepared in $25 \mathrm{~mm} \mathrm{~TB}, \mathrm{pH} 10$, with original concentrations of $0.5 \mathrm{~mm}$. Prior to analysis, they were mixed and diluted to suitable concentrations with $25 \mathrm{~mm}$ TB, $\mathrm{pH} 10$, unless noted.

\subsection{Optimization of PEO concentration}

New capillaries were treated with $0.5 \mathrm{M} \mathrm{NaOH}$ overnight. Prior to analysis, the base was flushed out with $400 \mathrm{~mm}$ TB buffer, $\mathrm{pH} 10$, by pressure means for $20 \mathrm{~s}$. Samples were injected into the capillary filled with TB buffers, $\mathrm{pH} 10$, in the anodic end by hydrodynamic injection at $30 \mathrm{~cm}$ height for $10 \mathrm{~s}$. After sample injection, the anodic end of the capillary was immersed in PEO solution that entered the capillary by electroosmotic flow (EOF) when applying an electric field of $375 \mathrm{~V} / \mathrm{cm}$. A number of PEO solutions prepared in $200 \mathrm{~mm}$ TB buffer, pH 9, with concentrations over a range of $0-2.0 \%$, were investigated for optimum conditions. After each run, the capillary was washed with $0.5 \mathrm{M} \mathrm{NaOH}$ at $1 \mathrm{kV}$ for $10 \mathrm{~min}$ to remove polymer solutions and refresh the capillary wall.

\subsection{On-line concentration and separation}

The on-line concentration method has been previously described [29]. Briefly, the capillaries were filled with $1 \mathrm{M}$ TB buffer, $\mathrm{pH} 10$, for better stacking and more rapid analysis. The samples were mixed and diluted with $25 \mathrm{~mm}$ TB 
<smiles>Nc1cccc2c(O)cccc12</smiles><smiles>Oc1cc(O)c2ccccc2c1</smiles>

5-A-1-N
1,3-DHN<smiles>O=Cc1cccc2ccccc12</smiles>

1-NAA<smiles>Oc1ccc2ccccc2c1</smiles>

2-NSA<smiles>[NH3+]c1ccc2c(O)cc(O)cc2c1</smiles>

7-A-4-H-2-NSA

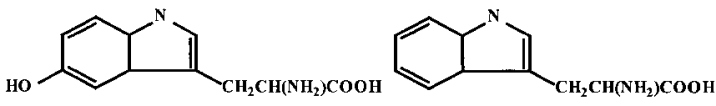

5-HT

L-Tryptophan

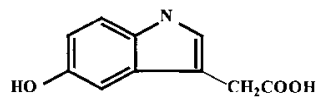

5-HIAA<smiles>Cc1cc(C#N)ccc1O</smiles>

HVA<smiles>CCCCc1ccc(O)c(OC)c1</smiles>

VMA

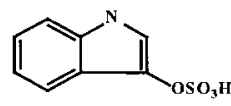

3-IXS

Figure 1. Chemical structures of the solutes analyzed in this work.

buffers, $\mathrm{pH} 10$, containing certain amounts of $\mathrm{NaCl}$. The effect of salt on stacking efficiency was tested in the range of $1-100 \mathrm{~mm}$. The samples were then injected at $30 \mathrm{~cm}$ height for 10-230 s and the separation was conducted at $375 \mathrm{~V} / \mathrm{cm}$. When the analytes migrating against EOF entered PEO solution, they slowed down and stacked due to decreases in the electrophoretic mobility. After each run, the capillary was treated as shown above.

\subsection{Urine and CSF}

The conditions used to perform the analysis of urine and CSF samples were similar to that described in Section 2.3 unless described. Without any pretreatment, the urine samples obtained from a normal male were directly injected at $30 \mathrm{~cm}$ height for certain times $(10-180 \mathrm{~s})$ to the capillary filled with 1 or $1.5 \mathrm{M}$ TB buffer, $\mathrm{pH} 10$. The on-line concentration and separation of the urine samples was conducted either in 2.0 or $1.5 \%$ PEO prepared in $200 \mathrm{~mm}$ TB buffer, $\mathrm{pH}$ 9. To determine the concentration of the markers, we spiked standards into the urine samples and the peak heights for the analytes were used for quantification. To increase the injection volume, a short plug of TP buffer consisting of Tris and propanic acid, $\mathrm{pH} 7$, was injected at $30 \mathrm{~cm}$ height for $10 \mathrm{~s}$ after injecting the urine sample. The CSF sample was collected from the L4-L5 interspace of an infant by lumbar puncture. Prior to analysis, the CSF sample was stored at $-80^{\circ} \mathrm{C}$. The CSF sample was directly injected in a $40 \mathrm{~cm}$ capillary filled with $1.5 \mathrm{~m}$ TB buffer, $\mathrm{pH} 10$, at $30 \mathrm{~cm}$ height for 10 or $80 \mathrm{~s}$ and subsequently the analysis was conducted at $15 \mathrm{kV}$ using 1.7\% PEO prepared in 200 mm TB buffer, pH 9.

\section{Results and discussion}

\subsection{Separation dependency of PEO}

There are only few examples showing separations of small solutes using polymer solutions in CE although they have addressed that the resolution increases due to interactions with polymer molecules [31-34]. To evaluate the effect of the polymer concentration on separating the five organic compounds (structures shown in Fig. 1), Figs. 2A-D were depicted, showing that a complete separation was only achieved in $2 \%$ PEO. Please note that the five peaks were not resolved in TB buffers (without $\mathrm{PEO}$ ) at $\mathrm{pH}$ values ranging from 8 to 10 . We reasoned that the increase in resolution is mostly due to increases in differences in migration times between any two adjacent analytes and possible interactions, such as hydrogen bonding and hydrophobic patches, with PEO molecules. A similar result has been shown in improved resolution due to hydrogen bonding between polyethylene glycol chains of the polymer and carboxylic groups or hydroxyl groups of small solutes [32]. As a result of increases in the viscosity and dynamic adsorption of PEO molecules on the capillary wall as PEO concentrations were increased, leading to small EOF mobilities, longer migration times 


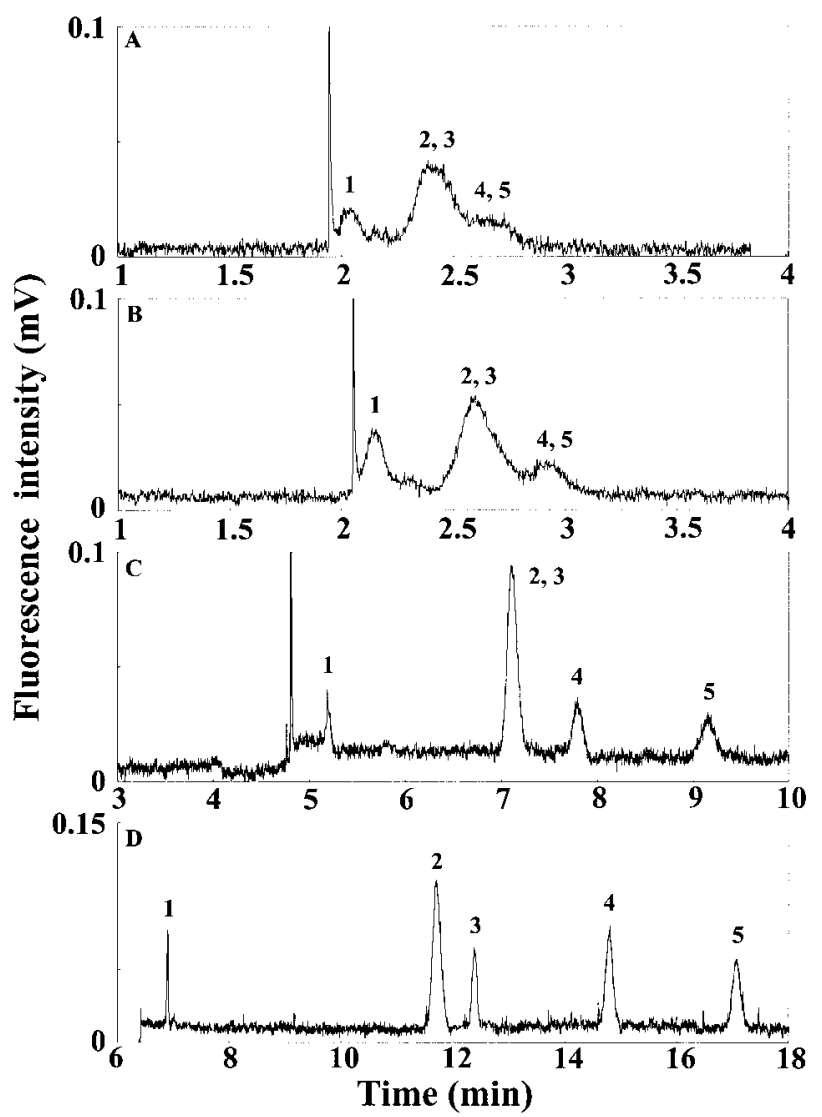

Figure 2. Effect of PEO solutions on separating five model organic solutes using a capillary filled with $400 \mathrm{~mm}$ TB buffers, $\mathrm{pH} 10$, at $15 \mathrm{kV}$. [PEO] $=0 \%$ in (A), $0.05 \%$ in (B), $1 \%$ in (C), $2 \%$ in (D). The PEO solutions were prepared in $200 \mathrm{~mm}$ TB buffers, $\mathrm{pH} 9$. Capillary, $40 \mathrm{~cm}$ in total length and $30 \mathrm{~cm}$ in effective length. Hydrodynamic injection was performed at $30 \mathrm{~cm}$ height for $10 \mathrm{~s}$. Peak identities (concentration): 1, 5-A-1-N (10 $\mu \mathrm{M}), 2,1,3-\mathrm{DHN}(0.2 \mathrm{~mm})$, 3, 1-NAA (15 $\mu \mathrm{M}), 4$, 2-NSA $(2 \mu \mathrm{M}), 5,7-\mathrm{A}-4-\mathrm{H}-2-\mathrm{NSA}$ $(30 \mu \mathrm{M})$.

were found as expected [35]. The relative standard deviation (RSD) for the migration times of 7-A-4-H-2-NSA was less than $2 \%$ using $2 \%$ PEO, implying that PEO adsorption did not cause serious memory effects. In other words, the use of $0.5 \mathrm{~m} \mathrm{NaOH}$ to wash the capillary after each run is effective to refresh the surface. Another benefit of using PEO solutions is that the sensitivity was further improved, supported by the fact that the LODs at a signal-to-noise ratio $(\mathrm{S} / \mathrm{N})=3$ for $5-\mathrm{A}-1-\mathrm{N}$ were 25 and $250 \mathrm{~nm}$ in $2 \%$ $\mathrm{PEO}$ and in TB buffer, respectively.

\subsection{Stacking efficiency}

Once PEO entered the capillary, the analytes with negative charges migrated into the more viscous zone. As a result, the electrophoretic mobility of the analytes de-

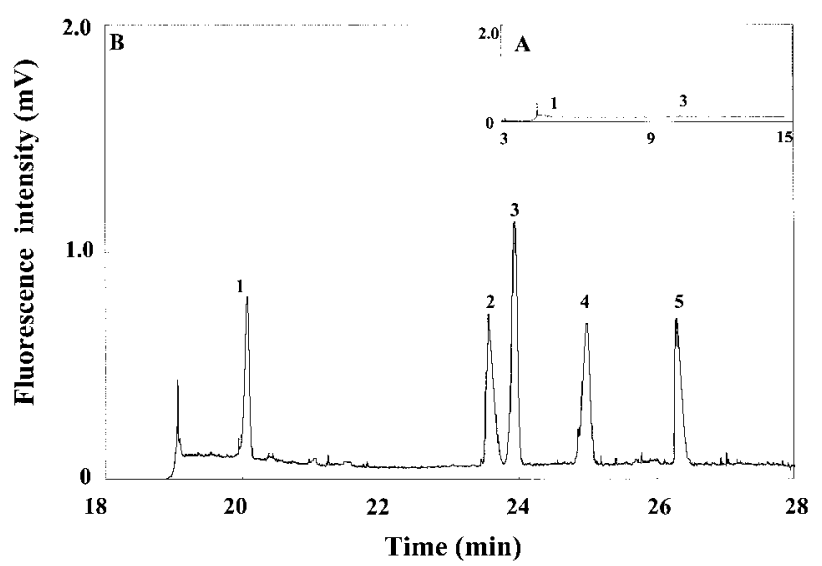

Figure 3. Analysis of five model organic solutes in the presence of EOF at $15 \mathrm{kV}$ using $2 \%$ PEO prepared in $200 \mathrm{~mm}$ TB solutions, $\mathrm{pH}$ 9. Hydrodynamic injections were performed at $30 \mathrm{~cm}$ height for $10 \mathrm{~s}$ in $(A)$ and $230 \mathrm{~s}$ in $(B)$, respectively. Peak identities (concentration): 1, 5-A1-N (0.5 $\mu \mathrm{m}), 2,1,3-\mathrm{DHN}(6.25 \mu \mathrm{M}), 3,1-\mathrm{NAA}(0.125 \mu \mathrm{M}), 4$, 2-NSA (50 nM); 5, 7-A-4-H-2-NSA (1 $\mu \mathrm{M})$. The capillary was filled with $1 \mathrm{~m}$ TB buffer, $\mathrm{pH} 10$. Other conditions were as in Fig. 2.

creased, resulting in stacking at the interface between the sample zone and PEO solution. On the basis of our knowledge learned from previous experiments [28-30, 36], instead of $400 \mathrm{~mm}$ TB, $1 \mathrm{~m}$ TB was used to fill the capillary for better stacking. Figure 3 clearly shows five resolved and sharp peaks when injecting at $30 \mathrm{~cm}$ height for $230 \mathrm{~s}$ (about $0.88 \mu \mathrm{L}$ ), indicating the analytes stacked. The linear regression coefficients for all model analytes in the injection range of 5-230 s were higher than 0.990 and the theoretical plates were higher than 0.77 million $/ \mathrm{m}$. As shown in our previous studies, the migration times increased with increasing injecting length because of greater PEO adsorption at low ionic strengths [29]. Please note that, without PEO solution, the peaks became extremely broad and the resolution was lost when injecting greater than $15 \mathrm{~s}$. The features of this new method are further tabulated in Table 1, which shows the LOD (S/N = 3) for 2-NSA was $30 \mathrm{pm}$, with a concentration factor (CF) of 333. Herein, CF is defined as the ratio of the LOD obtained by $5 \mathrm{~s}$ injection to that by $230 \mathrm{~s}$ injection under the same condition. For the solute $(5-A-1-N)$ with a less charge density and amino group, the stacking was more effective (1000-fold), indicating the existence of interactions with PEO molecules.

\subsection{Matrix effect}

As mentioned above that PEO adsorption decreased with increasing the ionic strength of the samples, a more rapid analysis could only be achieved at higher ionic strengths. 
Table 1. LOD values for the analytes achieved under different conditions

\begin{tabular}{lllllllll}
\hline \multicolumn{7}{c}{$\operatorname{LOD}(n=3)(\% R S D)$} \\
\hline 5-A-1-N & $\mu \mathrm{M}^{\mathrm{a}) \mathrm{b}) \mathrm{c})}$ & $\mathrm{nM}^{\mathrm{b}) \mathrm{c}) \mathrm{d})}$ & $\mathrm{nM}^{\mathrm{b}) \mathrm{d}) \mathrm{e})}$ & $\mathrm{CF}$ & $\mathrm{nM}^{\mathrm{d}) \mathrm{e}) \mathrm{f})}$ & $\mathrm{CF}$ & $\mathrm{nM}^{\mathrm{d}) \mathrm{e}) \mathrm{g})}$ & $\mathrm{CF}$ \\
1,3-DHN & $0.25(2.5)$ & $25.00(3.2)$ & $0.25(3.5)$ & 1000 & $0.50(3.2)$ & 500 & $30.00(3.2)$ & 8 \\
1-NAA & $1.50(3.2)$ & $300.00(3.8)$ & $4.00(4.3)$ & 375 & $10.00(3.9)$ & 150 & $15.00(3.7)$ & 100 \\
2-NSA & $0.025(2.8)$ & $5.00(2.9)$ & $0.09(4.5)$ & 278 & $0.05(4.0)$ & 500 & $0.06(3.7)$ & 417 \\
7-A-4-H-2-NSA & $0.01(3.5)$ & $2.50(3.7)$ & $0.03(4.3)$ & 333 & $0.01(4.2)$ & 1000 & $0.008(4.1)$ & 1250 \\
& $1.00(3.9)$ & $500.00(3.2)$ & $10.00(4.7)$ & 100 & $6.00(4.5)$ & 167 & $12.00(4.3)$ & 83 \\
\hline
\end{tabular}

a) The separation was conducted in $400 \mathrm{~mm} \mathrm{~TB}, \mathrm{pH} 10$.

b) Samples were dissolved in $25 \mathrm{~mm}$ TB buffers, $\mathrm{pH} 10$.

c) Samples were injected at $30 \mathrm{~cm}$ height for $5 \mathrm{~s}$.

d) The separation was conducted in 2\% $\mathrm{PEO}, \mathrm{pH}$, using the capillary filled with $1 \mathrm{~m}$ TB buffer, $\mathrm{pH} 10$.

e) Samples were injected at $30 \mathrm{~cm}$ height for $230 \mathrm{~s}$.

f) Samples were dissolved in $25 \mathrm{~mm}$ TB buffers, $\mathrm{pH}$ 10, containing $10 \mathrm{~mm} \mathrm{NaCl}$.

g) Samples were dissolved in 25 mм TB buffers, pH 10, containing $100 \mathrm{~mm} \mathrm{NaCl}$.

Table 2. Salt impact on the peak height $(h)$, migration time $(t)$, and peak width $(w)$

\begin{tabular}{|c|c|c|c|c|c|c|c|c|c|c|c|c|c|}
\hline & & \multicolumn{12}{|c|}{$\mathrm{NaCl}(\mathrm{mm})$} \\
\hline & & \multicolumn{3}{|c|}{$0^{\mathrm{a})}$} & \multicolumn{3}{|c|}{$1^{\mathrm{a})}$} & \multicolumn{3}{|c|}{$10^{\mathrm{a})}$} & \multicolumn{3}{|c|}{$100^{b)}$} \\
\hline & $\begin{array}{l}\text { Concentra- } \\
\text { tion }(\mu \mathrm{m})\end{array}$ & $\begin{array}{l}h \\
(\mu \mathrm{V})\end{array}$ & $\begin{array}{l}t \\
(\min )\end{array}$ & $\begin{array}{l}w \\
(\min )\end{array}$ & $\begin{array}{l}h \\
(\mu \mathrm{V})\end{array}$ & $\begin{array}{l}t \\
(\min )\end{array}$ & $\begin{array}{l}w \\
(\min )\end{array}$ & $\begin{array}{l}h \\
(\mu \mathrm{V})\end{array}$ & $\begin{array}{l}t \\
(\min )\end{array}$ & $\begin{array}{l}w \\
(\min )\end{array}$ & $\begin{array}{l}h \\
(\mu \mathrm{V})\end{array}$ & $\begin{array}{l}t \\
(\min )\end{array}$ & $\begin{array}{l}w \\
(\min )\end{array}$ \\
\hline $5-A-1-N$ & 0.5 & 710 & 20.07 & 0.07 & 857 & 17.55 & 0.07 & 446 & 14.63 & 0.17 & 12 & 6.55 & 0.15 \\
\hline 1,3-DHN & 6.25 & 615 & 23.56 & 0.20 & 368 & 22.15 & 0.15 & 430 & 18.95 & 0.08 & 257 & 11.67 & 0.22 \\
\hline 1-NAA & 0.125 & 1073 & 23.94 & 0.22 & 1362 & 22.59 & 0.12 & 1778 & 19.42 & 0.08 & 1438 & 12.50 & 0.09 \\
\hline 2-NSA & 0.05 & 615 & 24.98 & 0.25 & 664 & 24.36 & 0.17 & 969 & 20.94 & 0.13 & 1115 & 14.89 & 0.14 \\
\hline 7-A-4-H-2-NSA & 1 & 644 & 26.28 & 0.22 & 602 & 26.52 & 0.18 & 679 & 22.13 & 0.14 & 346 & 16.49 & 0.21 \\
\hline
\end{tabular}

a) Samples were injected at $30 \mathrm{~cm}$ height for $230 \mathrm{~s}$; other conditions were as in Fig. 3.

b) Samples were injected at $30 \mathrm{~cm}$ height for $120 \mathrm{~s}$.

Table 2 shows the migration times for 7-A-4-H-2-NSA were $26.28 \mathrm{~min}$ and $22.13 \mathrm{~min}$ when the samples were prepared in $25 \mathrm{~mm}$ TB buffers without and with containing $10 \mathrm{~mm} \mathrm{NaCl}$, respectively. Although it has been shown that peak broadening occurred due to Joule heating and poor stacking when injecting sample prepared in high-conductivity media [23], the impact was not marked, mainly because the diffusion was less profound and stacking took place in viscous PEO solution. Apparently, Table 2 does not show a clear trend of the salt impact on the fluorescence intensity. It is presumably because not only did salts affect stacking efficiency, but also affected the quantum yields of some analytes [37]. For 2-NSA, we have found that the peak height increased (peak width decreased) with increasing the salt concentration. This is due to less diffusion problems (short migration time) and weaker interactions with the capillary wall at high salts. However, an opposite result was found in 5-A-1-N containing an amino group, mostly due to salt quenching, supported by a similar result shown in 7-A-4-H-2-NSA.
Table 1 further shows the LOD for 2-NSA was 10 pм when injecting $0.88 \mu \mathrm{L}$ of the sample prepared in $25 \mathrm{~mm}$ TB buffers containing $10 \mathrm{~mm} \mathrm{NaCl}$, with reasonable reproducibility. The RSD values of the migration time and the peak heights for all solutes were less than $2 \%$ and $4.7 \%$, respectively. Strikingly, the LOD for 2-NSA was $8 \mathrm{pm}$ when injecting $0.43 \mu \mathrm{L}$ of the sample prepared in $25 \mathrm{~mm}$ TB buffers containing $100 \mathrm{~mm} \mathrm{NaCl}$. It is noteworthy that the sensitivity was improved by 1250 -fold compared to that obtained in TB free buffer by a hydrodynamic injection at $30 \mathrm{~cm}$ height for $5 \mathrm{~s}$. We suspected that sample self-stacking (chloride) was also a contributor for high sensitivity [38]. However, an effort made to improve sensitivity by injecting greater sample volumes was failed, mostly because of Joule heating.

\subsection{Separation of indoles}

As mentioned in Section 1, this work also aimed to develop a method with the potential for metabolomics. To test the possibility, we started from applying the devel- 


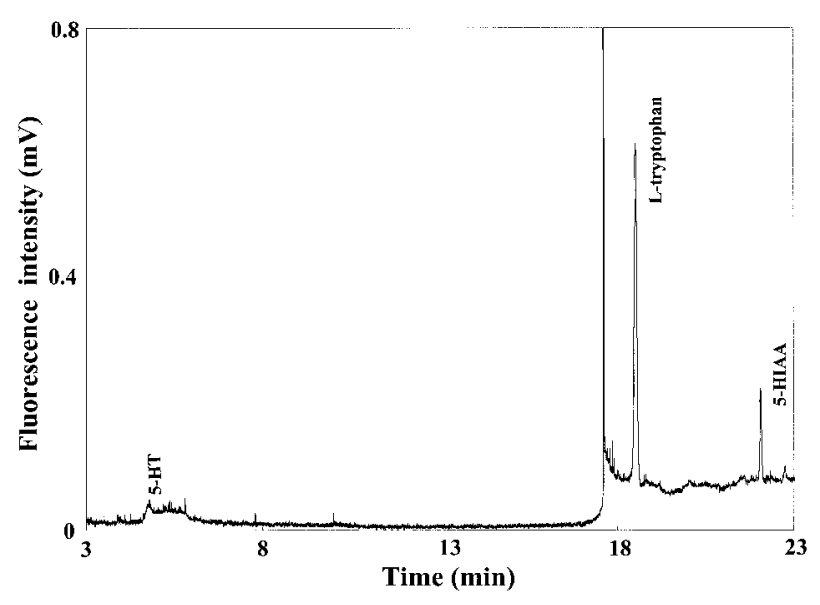

Figure 4. Analysis of 5-HT, L-tryptophan, and 5-HIAA in the presence of EOF at $15 \mathrm{kV}$ using $2 \% \mathrm{PEO}, \mathrm{pH} 9$. $[5-\mathrm{HT}]=10 \mathrm{~nm}$, [L-tryptophan] $=20 \mathrm{~nm}$, and [5-HIAA $]=$ $10 \mathrm{~nm}$. Hydrodynamic injection was performed at $30 \mathrm{~cm}$ height for $230 \mathrm{~s}$. Other conditions were as in Fig. 3.

oped method for the analysis of a sample containing Ltryptophan, 5- $\mathrm{HT}$, and 5-HIAA that are heterocyclic amine compounds and present in cells and extracellular fluids of living organisms. The determination of these analytes in nervous tissues, blood, and urine is of considerable importance and interest because they are neurotransmitters and are markers associated with many diseases, such as physiology of mental illness and neurological disorders [39]. Figure 4 depicts the separation of 5-HT, Ltryptophan, and 5-HIAA with hydrodynamic injection at $30 \mathrm{~cm}$ height for $230 \mathrm{~s}$. Since 5-HT, with partial positive charges, did not enter the PEO zone, and thus did not stack well at the interface between the sample zone and PEO solution. As a result, band broadening occurred when injecting for more than $60 \mathrm{~s}$. The LOD values for $5-$ HT were $20 \mathrm{~nm}$ and $4 \mathrm{~nm}$, when hydrodynamic injections were carried out at $30 \mathrm{~cm}$ height for 5 and $60 \mathrm{~s}$, respectively. It should be pointed out that stacking still occurred due to a field amplification and $\mathrm{pH}$ junction. On the other hand, L-tryptophan and 5-HIAA entered the PEO solution and did stack up to $230 \mathrm{~s}$ injection, resulting in the LOD values down to 70 and $90 \mathrm{pm}$, respectively. The sensitivity improvement for L-tryptophan was about 300-fold compared to that in TB buffer by conventional injection (5 s). The linear relationships between the peak heights for Ltryptophan and 5-HIAA and injection times were found, with $R^{2}=0.995$ and 0.985 , respectively. With the LOD less than $\mathrm{nm}$, it is thought that this new method is useful for analysis of biological samples. In comparison to $\mathrm{CW}$ UV argon-ion laser, Nd:YAG laser provides about 10 times higher LOD (tens to hundreds nM) for amines and acids in solutions without PEO, but it is much cheaper and almost

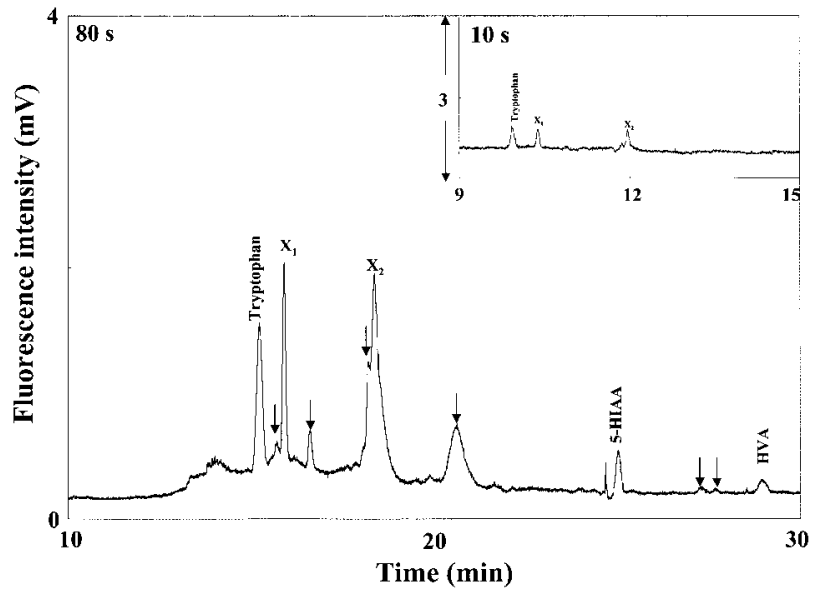

Figure 5. Analysis of a CSF sample in the presence of EOF at $15 \mathrm{kV}$ using 1.7\% PEO, pH 9. The capillary was filled with $1.5 \mathrm{M} \mathrm{TB}, \mathrm{pH} 10$. The injections of a CSF sample were conducted at $30 \mathrm{~cm}$ height for $10 \mathrm{~s}$ in the upper panel and $80 \mathrm{~s}$ in the main frame, respectively. $X_{1}$ and $X_{2}$ are two unidentified peaks. Peaks marked as arrow bars were only detected when performing on-line concentration. Other conditions were as in Fig. 3.

free from maintenance. Although the developed on-line concentration method is simple, free from contamination, and sensitive when compared with derivatization methods, it is only suitable for analytes with intrinsic fluorescence characteristics.

\subsection{Analysis of CSF}

The measurement of metabolites HVA and 5-HIAA in CSF has received great attention for indirect accesses of the central nervous system activity of dopamine and serotonin in schizophrenia research for approximately two decades [40]. Without stacking, only three peaks were detected as shown in the upper panel of Fig. 5. The first peak in the electropherogram is L-tryptophan, and the other two possibly correspond to proteins, such as $\beta$ trace protein and HSA (not identified). By applying this concentration technique, more peaks, including 5-HIAA and HVA, were detected as shown in Fig. 5. The concentrations for 5-HIAA and HVA were 0.48 and $2.7 \mu \mathrm{M}$, with RSD values ( $n=3$ ) of 3.5 and $2.5 \%$, respectively. Because comparisons of the differential ratio of HVA/HIAA and different fingerprints of the electropherograms between healthy control and medication-free schizophrenic patients are important from a practical point of view, the result shows potential for clinics. 
Table 3. The biological relevance and concentration of five markers in urine

\begin{tabular}{|c|c|c|c|c|}
\hline Compound & $\begin{array}{l}\text { Normal } \\
\text { (evela) }\end{array}$ & Biological relevance & Linear regression $\left.{ }^{b}\right)$ & $\begin{array}{l}\text { Urinary concen- } \\
\text { tration }(\mu \mathrm{M})\end{array}$ \\
\hline 5-HIAA & $\begin{array}{l}2 \mathrm{mg} / \mathrm{day} \\
\quad(<50 \mu \mathrm{M})\end{array}$ & Carcinoid tumor screening & $\begin{array}{l}Y=2568.7 X+940.63 \\
R^{2}=0.9940\end{array}$ & $36.6(3.6)^{\mathrm{c})}$ \\
\hline HVA & $\begin{array}{l}6 \mathrm{mg} / \text { day } \\
\quad(<40 \mu \mathrm{m})\end{array}$ & $\begin{array}{l}\text { Neuroblastoma and hepatic } \\
\text { encephalopathy screening }\end{array}$ & $\begin{array}{l}Y=21.826 X+64.558 \\
R^{2}=0.9944\end{array}$ & $29.5(2.4)$ \\
\hline VMA & $\begin{array}{l}3.5 \mathrm{mg} / \text { day } \\
(<45 \mu \mathrm{M})\end{array}$ & $\begin{array}{l}\text { Neuroblastoma and hepatic } \\
\text { encephalopathy screening }\end{array}$ & $\begin{array}{l}Y=27.845 X+81.298 \\
R^{2}=0.9932\end{array}$ & $29.1(1.4)$ \\
\hline HSA & $\begin{array}{r}150 \mathrm{mg} / \text { day } \\
(<1.5 \mu \mathrm{M})\end{array}$ & $\begin{array}{l}\text { Sickle cell, renal tubular screening, } \\
\text { and nephrosclerosis screening }\end{array}$ & $\begin{array}{l}Y=107.9 X+120.08 \\
R^{2}=0.9974\end{array}$ & $1.11(1.5)$ \\
\hline $5-\mathrm{HT}$ & $\begin{array}{l}146 \mu \mathrm{g} / \text { day } \\
(<0.9 \mu \mathrm{M})\end{array}$ & Pheochromocytoma screening & $\begin{array}{l}Y=361.24 X+64.423 \\
\quad R^{2}=0.9974\end{array}$ & $0.17(3.1)$ \\
\hline
\end{tabular}

a) From Ref. [9]

b) $Y$ represents peak height $(\mu \mathrm{V})$ and $X$ represents the concentration of spiked analytes.

c) $\% \operatorname{RSD}(n=3)$

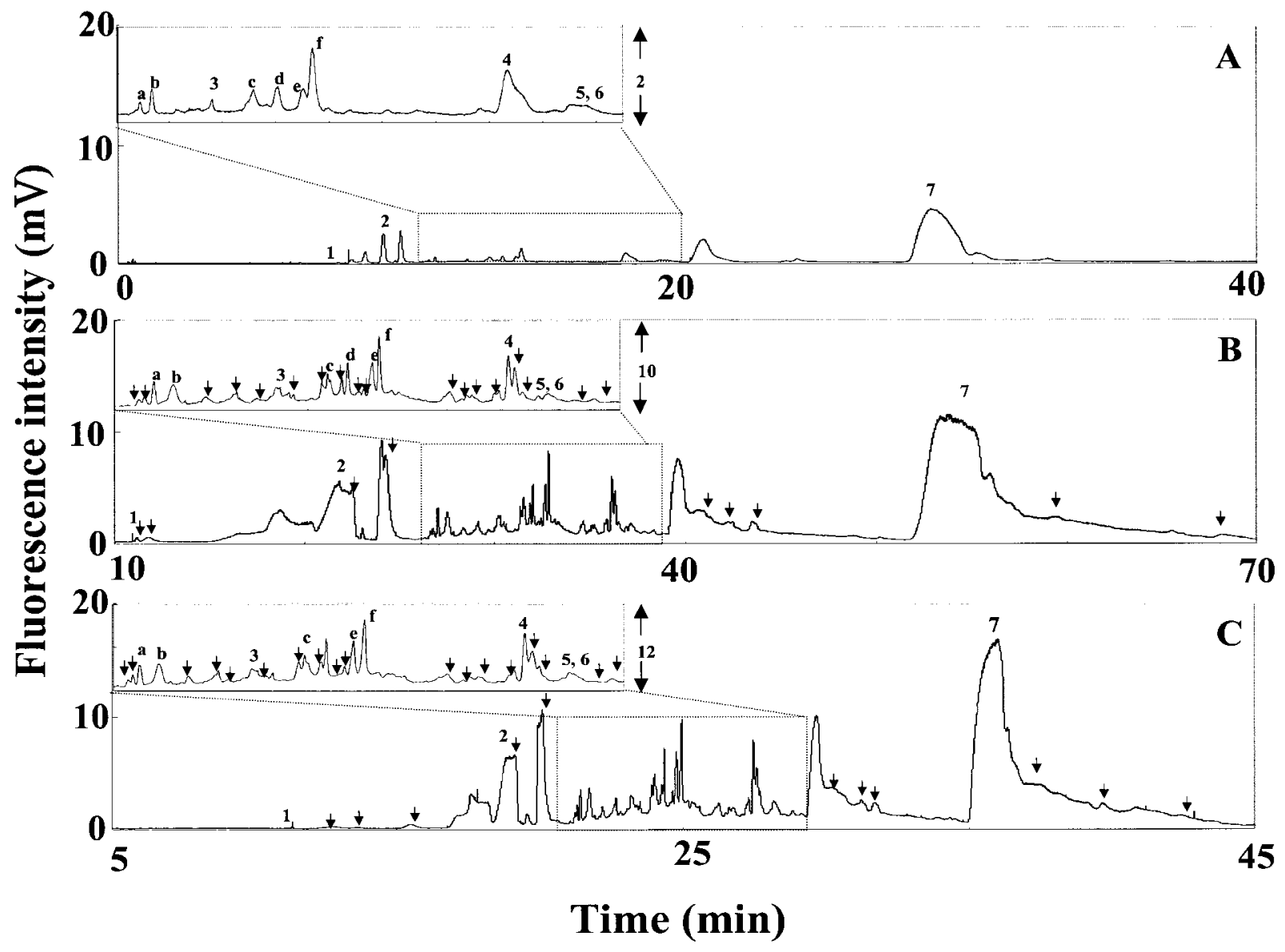

Figure 6. Analysis of a urine sample in the presence of EOF at $15 \mathrm{kV}$ using 1.5\% PEO, pH 9. Peak identities: 1, 5- HT; 2, L-tryptophan; 3, HSA; 4, 5-HIAA; 5, HVA; 6, VMA; 7, 3-IXS; a-f represent unidentified peaks. The $60 \mathrm{~cm}$ capillary was filled with $1.5 \mathrm{~m} \mathrm{~TB}, \mathrm{pH} 10$. The injections of a urine sample were conducted at $30 \mathrm{~cm}$ height for $10 \mathrm{~s}$ in (A), and $180 \mathrm{~s}$ in $(\mathrm{B})$ and $(\mathrm{C})$, respectively. A short plug of TP buffer was injected at $30 \mathrm{~cm}$ height for $10 \mathrm{~s}$ after sample injection in (C). Other conditions were as in Fig. 3. 


\subsection{Analysis of urine with a short plug of low-pH buffer}

Scientists have made great efforts to determine HVA and VMA, the major metabolites of catecholamines, in urine for neurologic diagnosis and for monitoring the response of therapy in illnesses such as neuroblastoma and phaeochromocytoma [41]. Without stacking, numerous peaks including seven important markers that have been identified were detected as shown in Fig. 6A. On the basis of the peak heights and the regression lines shown in Table 3 , the concentrations of five analytes in urine were calculated, which were in good agreement with reported data [42]. Without any sample pretreatment, the RSD values for the quantitative results of five solutes were less than $3.6 \%$, indicating that this method is rugged and simple. Compared to Fig. 6A, more peaks were detected as shown in Fig. 6B when injecting greater volumes of the sample, indicating that stacking did occur. Since we used a UV laser as the light source, the new peaks should correspond to solutes with native fluorescence in the UV range, including benzoic compounds, polycyclic aromatic hydrocarbons, heterocyclic compounds, peptides containing tryptophan, tyrosine, and phenylalanine residues, nucleotides, oligonucleotides, and so on. By using unidentified peaks marked as a-f and identified peaks marked as $1-7$ as standards, we were able to identify some new peaks (arrow bars). Although more peaks were detected when injecting greater volumes, the loss of resolution was found, especially for those with high concentrations. Another shortage of this method is the analysis was slow (the migration time for 3-IXS was $53.78 \mathrm{~min})$. In order to shorten separation time and optimize resolution, we injected a short plug of TP buffer $(\mathrm{pH} 7)$ after sample injection. Apparently, Fig. 6C shows that the migration time for $3-I X S$ is shorter (35.98 vs. 53.78 in Fig. 6B) and some peaks (e.g., peaks 2 and 7) are sharper. This is due to better stacking and reduced adsorption of the urine matrices and PEO, presumably because of large amounts of propanate in the short plug. The plug also caused solutes to migrate further apart from small ions like chloride, thereby reducing the effect of salt on stacking and peak profiles. In comparison with results obtained by $10 \mathrm{~s}$ injection ( $40 \mathrm{~cm}$ capillary), the heights of peaks a-f were enhanced by as many as 51 -fold when injecting for $180 \mathrm{~s}$ with the short plug of TP buffer. More importantly, by applying a short plug of TP buffer, 52 peaks were detected in $35.98 \mathrm{~min}$ (the migration time for $3-\mathrm{IXS}$ ).

\section{Concluding remarks}

We have developed methods for increasing sensitivity and selectivity. The selectivity for the small solutes has been optimized in the presence of PEO, mainly because of reduced band broadening and possible interactions between the analytes and PEO molecules. On the basis of the reduced electrophoretic mobility of the analytes in PEO solution, this method allows injection of samples up to $0.88 \mu \mathrm{L}$, with a 1250 -fold improvement in the sensitivity for 2-NSA. The LOD values were $8 \mathrm{pm}$ for 2-NSA, $70 \mathrm{pm}$ for L-tryptophan, and $90 \mathrm{pm}$ for 5-HIAA. With such low LODs, this method has been demonstrated for the analysis of urine and CSF. It should be noted that salts at optimum concentrations play a significant role in increasing stacking efficiency and reducing PEO adsorption on one hand, deteriorate fluorescence for some analytes on the other hand.

We also report a method for optimum resolution, migration time and sensitivity for the analysis of large-volume urine samples through injection of a short plug of low-pH buffer. The mobility of solutes further reduces when entering the low-pH zone, thereby allowing a greater volume of urine samples injected. As a result, the peak-height enhancements for some unidentified solutes are up to 51-fold and more peaks were detected. Although it is impossible and not our goal to identify all these peaks, we believe that more peaks should be identified if we combine this method with mass spectrometry, which should be useful for neurochemistry and metabolomics. In the future, our effort will be made to obtain important information by comparing the electropherograms of biological samples, such as urine and CSF, from normal people and unhealthy people or from people with and without taking drugs like dopamine.

This work was supported by the National Science Council of the Republic of China under contract number NSC 902113-M002-052.

Received October 15, 2001

\section{References}

[1] Cash, P., Electrophoresis 2000, 21, 1187-1201.

[2] Hanash, S. M., Electrophoresis 2000, 21, 1202-1209.

[3] Raamsdonk, L. M., Teusink, B., Broadhurst, D., Zhang, N., Hayes, A., Walsh, M. C., Berden, J. A., Brindle, K. M., Kell, D. B., Rowland, J. J., Westerhoff, H. V., Dam, K. V., Oliver, S. G., Nat. Biotechnol. 2001, 19, 45-50.

[4] Han, F., Huynh, B. H., Shi, H., Lin, B., Ma, Y., Anal. Chem. 1999, 71, 1265-1269.

[5] Hsieh, M.-M., Kuo, Y.-C., Lyu, M.-J., Chang, H.-T., J. Chromatogr. A 2000, 898, 133-139.

[6] Melason, J. E., Boulet, C. A., Lucy, C. A., Anal. Chem. 2001, 73, 1809-1813.

[7] Wallingford R. A., Ewing A. G., J. Chromatogr. 1988, 441, 299-309.

[8] Thorsén, G., Engström, A., Josefsson, B., J. Chromatogr. A 1997, 786, 347-354. 
[9] Paquette, D. M., Sing, R., Banks, P. R., Waldron, K. C. J. Chromatogr. B 1998, 714, 47-57.

[10] Chan, K. C., Muschik, G. M., Issaq, H. J., Electrophoresis 2000, 21, 2062-2066.

[11] Kuijt, J., García-Ruiz, C., Stroomberg, G. J., Marina, M. L., Ariese, F., J. Chromatogr. A 2001, 907, 291-299.

[12] Zaugg, S., Zhang, X., Sweedler, J., Thormann, W., J. Chromatogr. B 2001, 752, 17-31.

[13] Chen, Z., Wu, J., Baker, G. B., Parent, M., Dovichi, N. J., J. Chromatogr. A 2001, 914, 293-298.

[14] Hadwiger, M. E., Torchia, S. R., Park, S., Biggin, M. E., Lunte, C. E., J. Chromatogr. B 1996, 681, 241-249.

[15] He, Y., Lee, H. K., Anal. Chem. 1999, 71, 995-1001.

[16] Xiong, Y., Park, S.-R., Swerdlow, H., Anal. Chem. 1998, 70, 3605-3611.

[17] Palmer, J., Burgi, D. S., Munro, N. J., Landers, J. P., Anal. Chem. 2001, 73, 725-731.

[18] Chien, R.-L., Helmer, J. C., Anal. Chem. 1991, 63, 13541361.

[19] Timerbaev, A. R., Fukushi, K., Miyado, T., Ishio, N., Saito, K., Motomizu, S., J. Chromatogr. A 2000, 888, 309-319.

[20] Danková, M., Kaniansky, D., Fanali S., Iványi, F., J. Chromatogr. A 1999, 838, 31-43.

[21] Chen, S., Lee, M. L., Anal. Chem. 2000, 72, 816-820.

[22] Shihabi, Z. K., J. Chromatogr. A 1996, 744, 231-240.

[23] Zhao, Y., Lunte, C. E., Anal. Chem. 1999, 71, 3985-3991.

[24] Quirino, J. P., Terabe, S., Boček, P., Anal. Chem. 2000, 72, 1934-1940.

[25] Palmer, J., Landers, J. P., Anal. Chem. 2000, 72, 1941-1943.

[26] Britz-Mackibbin, P., Chen, D. D. Y., Anal. Chem. 2000, 72, 1242-1252.
[27] Weiss, D. J., Saunders, K., Lunte, C. E., Electrophoresis 2001, 22, 59-65.

[28] Hsieh, M.-M., Tseng, W.-L., Chang, H.-T., Electrophoresis 2000, 21, 2904-2910.

[29] Tseng, W.-L., Chang, H.-T., Anal. Chem. 2000, 72, 48054811.

[30] Huang, M.-F., Hsu, C.-E., Tseng, W.-L., Lin, Y.-C., Chang, H.T., Electrophoresis 2001, 22, 2281-2290.

[31] Soini, H., Riekkola, M. L., Novotny, M. V., J. Chromatogr. A 1994, 680, 623-634.

[32] Esaka, Y., Yamaguchi, Y., Kano, K., Goto, M., Anal. Chem. 1994, 66, 2441-2445.

[33] Bednár, P., Stránský, Z., Barták, P., Adamovský, P., J. Chromatogr. A 1999, 838, 89-99.

[34] Shimizu, T., Kenndler, E., Electrophoresis 1999, 20, 33643372.

[35] Tseng, W.-L., Hsieh, M.-M., Wang, S.-J., Chang, H.-T., J. Chromatogr. A 2000, 894, 219-230.

[36] Tseng, W.-L., Chang, H.-T., Electrophoresis 2001, 22, 763770.

[37] Chang, H.-T., Yeung, E. S., Anal. Chem. 1995, 67, 10791083.

[38] Beckers, J. L., Boček, P., Electrophoresis 2000, 21, 27472767.

[39] Chen, D.-C., Zhan, D.-Z., Cheng, C.-W., Liu, A.-C., Chen, C. H., J. Chromatogr. A 2001, 750, 33-39.

[40] Wieselgren, I. M., Lindström, L. H., Psychiat. Res. 1998, 81, 101-110.

[41] García, A., Heinänen, M., Jiménez, L. M., Barbas, C., J. Chromatogr. A 2000, 871, 341-350.

[42] Panholzer, T. J., Beyer, J., Lichtwald, K., Clin. Chem. 1999, 45, 262-268. 